\title{
Low Distortion Delaunay Embedding of Trees in Hyperbolic Plane
}

\author{
Rik Sarkar \\ Institut Für Informatik, \\ Freie Universität Berlin, Germany \\ sarkar@inf .fu-berlin.de
}

\begin{abstract}
This paper considers the problem of embedding trees into the hyperbolic plane. We show that any tree can be realized as the Delaunay graph of its embedded vertices. Particularly, a weighted tree can be embedded such that the weight on each edge is realized as the hyperbolic distance between its embedded vertices. Thus the embedding preserves the metric information of the tree along with its topology. The distance distortion between non adjacent vertices can be made arbitrarily small - less than a $(1+\varepsilon)$ factor for any given $\varepsilon$. Existing results on low distortion of embedding discrete metrics into trees carry over to hyperbolic metric through this result. The Delaunay character implies useful properties such as guaranteed greedy routing and realization as minimum spanning trees.
\end{abstract}

\section{Introduction}

Embedding given data into a standard space lets us use properties of the target space as additional structure in the original dataset, and brings to front information that is hard to detect in the raw input. If the target space is Euclidean, that allows us to visualize and treat the data geometrically. In general, if it is a metric space, the properties of the metric can aid in understanding the original data and answering queries. This approach has been found relevant to a variety of subjects such as data visualization, network analysis, routing, localization, machine learning, statistics, biology and many others.

Trees are an important class of data structures. They occur commonly in natural scenarios, therefore associating trees with geometric spaces can be of benefit in many domains. Realizing trees as Delaunay graphs lets us combine the structural properties of trees with those of Delaunay graphs as well as those of the ambient space.

In this paper, we show that this can always be achieved in the hyperbolic spaces. In particular, we tackle the question for weighted trees and show that any given edge weights can be realized exactly (upto a choice of unit) in the delaunay embedding, while keeping the overall distortion arbitrarily low. 


\subsection{Related Work}

Trees in Euclidean Plane. Monma and Suri [1] address embedding minimum spanning trees in the Euclidean space. They analyze questions of perturbations to the embedding and their effect on the topology of the MST. Relevant to us, they consider the problem of which trees can be realized as minimum spanning tree in the Euclidean plane, and show that any tree with maximum vertex degree of 5 or less admits an embedding as a minimum spanning tree. The topic of distortion of a tree embedded in euclidean metric is analyzed in [10].

Low Distortion Metric Embeddings. This is an extensively studied subject that we do not have the space to discuss. We just note that metrics can have nice probabilistic embeddings into trees. $N$-point metrics have distributions over embeddings in trees, and weighted graphs have distributions over spanning trees with small expected distortions. See [6/34].

The hyperbolic metric behaves like a tree in many respects. One way to model this is the concept of $\delta$-hyperbolic metric [8]. Chepoi and co-authors 1 show that a graph that has an $n$-node $\delta$-hyperbolic graph admits an approximating tree of additive error $O(\delta \log n)$.

Hyperbolic Embeddings Embedding graphs into hyperbolic spaces can provide advantages that we do not get in Euclidean spaces or trees. Kleinberg [9] considers the problem of greedy routing in wireless networks. This is the method, where a node routes a message by forwarding it to the neighbor that is nearest to the destination. The idea in [9] is to embed a spanning tree of the network into the hyperbolic plane such that this routing always works successfully on the tree, and thereby on the original network. Eppstein and Goodrich [5] show a related method that uses small sized coordinates. Cvetkovski et al. [2] extend [9] to incorporate dynamic insertion of edges. Papadopoulos et al. 12 show that hyperbolic embedding can naturally give rise to scale free networks and as before, can support greedy routing. Zeng et al. [15] embed the universal covering space of a network into the hyperbolic plane as a Riemann surface and show that this can be used to easily find paths of different homotopy types in the network.

\subsection{Our Contributions}

The choice of the target space determines the properties we can expect to obtain from the embedding. In this paper, we use the hyperbolic plane as the standard target space and show that this works remarkably well for all kinds of trees weighted as well as unweighted, and induces many desirable features. We present the important ideas and theorems in the main body of the paper. Details of proofs can be found in the full version of the paper online [13.

Delaunay Embedding. Delaunay graphs are known to have many useful properties. If a graph can be treated as the Delaunay graph in some space, we can expect to leverage some of these properties for our purposes. With this motivation, we take up the question of realizing trees as Delaunay graphs, and show 
that the vertices of any tree can be embedded in the hyperbolic plane such that their Delaunay graph is the original tree. This leads to two immediate consequences: Realization of any tree as the minimum spanning tree of its vertices and guaranteed delivery by greedy routing. The first overcomes the constant degree bound of Euclidean case, the second implies the result of [9]. The embedding in [9] is a specific Delaunay embedding based on tiling of $\mathbb{H}$ by ideal regular $d$-gons, where $d$ is the max degree. We generalize this to not depend on a tiling, which gives greater flexibility needed to create the low distortion embeddings in sections 4 and 5 .

Weighted Trees. Suppose the given tree has weights or lengths defined for its edges. This induces a metric and we refer to the input as a metric tree. We show that the vertices of any such metric tree has a Delaunay embedding in the hyperbolic plane, such that each Delaunay edge has a length that is the multiple of the edge's prescribed length by a constant. As before, the properties implied by Delaunay embedding (MST and greedy routing) are retained.

Thus, the embedding not only preserves the topology of the tree, it also preserves the geometry - the metric information from the tree. The effect of the constant is negligible: since it is same globally, once it has been computed we can scale our unit of length to eliminate the factor. Our definition of distortion is oblivious to such global scaling.

Distortion of Metric Trees. While we preserve edge lengths precisely, the overall metric may not be preserved. The hyperbolic distance between $v_{i}$ and $v_{j}$ will be less than or equal to the path length between the two vertices in the tree. We show that this distortion can be kept arbitrarily low. Given any $\varepsilon$, we show it is possible to do the Delaunay embedding such that the distortion is less than $1+\varepsilon$. Thus the tree can be seen as a hyperbolic spanner of the embedded vertices.

This implies distortion bounds for embedding of metrics in general. The results in 6 6.34 show low distortion bounds (probabilistic and average) for embedding arbitrary $n$ point metrics into trees. It is therefore possible to embed arbitrary metrics into hyperbolic metric with the same bounds, using the tree embedding as an intermediate step.

\section{Basics and Notations}

This section introduces some basic facts and notations about the geometry of hyperbolic plane that will be used later. We denote the hyperbolic plane by $\mathbb{H}$. For a more detailed exposition of these ideas, see [7. We present the ideas in terms of the plane $\mathbb{H}$, but they generalize directly to higher dimensions. We use $|\cdot|_{\mathbb{H}}$ to denote distances in hyperbolic metric. Correspondingly, we use $|\cdot|_{T}$ to denote distances in the metric of the input tree.

In hyperbolic geometry, the axioms of euclidean geometry are all true, except the parallel axiom, which is replaced by the Hyperbolic Axiom: There is a line $l$ and point $P$ not on $l$, such that there are at least 2 different lines through $P$ parallel to $l$. 
This leads to the general property that given a line, there are an infinite number of different lines parallel to it through an outside point. Parallel lines can be of different types. A pair of parallel lines are said to be limiting parallel if they approach each-other asymptotically without intersecting. Such a pair does not admit a common line perpendicular to both, and the distance between the two does not have a minimum. A pair of parallel lines are called divergent parallel if they move away from each-other in both directions. They have a common segment perpendicular to both. This segment achieves the minimum distance between the two lines.

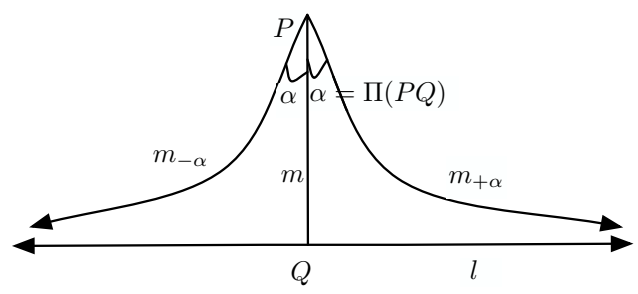

Fig. 1. Line $m \perp l$ and rays $m_{-\alpha}$ and $m_{+\alpha}$ are limiting parallel to $l$. The angle $\alpha$ depends on the length of the segment $P Q$. Hyperbolic straight lines $m_{-\alpha}$ and $m_{+\alpha}$ look curved because our figure is in euclidean space.

Given a line $l$ and a point $P$ outside, there is always a point $Q$ on $l$ such that $P Q \perp l$. Through $P$, there are always rays $m_{+\alpha}$ and $m_{-\alpha}$ that are limiting parallel to $l$ in the two directions. The angle between $P Q$ and $m_{+\alpha}$ (or symmetrically, the angle between $P Q$ and $m_{-\alpha}$ ) is called the angle of parallelism and represented by $\Pi(P Q)$. The Bolyai and Lobachevsky formula gives its value in terms of the length $|P Q|_{\mathbb{H}}$ :

$$
\tan \frac{\Pi(P Q)}{2}=e^{-|P Q|_{\mathbb{H}} / k},
$$

where $k$ is a constant for the hyperbolic plane in consideration. Note that $\Pi(P Q)$ is always less than $\pi / 2$ radians, since $P Q$ cannot be perpendicular to a ray that is limiting parallel to $l$. Given $l$ and $P$, the limiting parallel rays and the angle $\Pi(P Q)$ are unique. A ray that creates a larger angle with $P Q$ will be divergent parallel, while a ray with smaller angle will intersect $l$.

The region bounded by rays $m_{-\alpha}$ and $m_{+\alpha}$ and containing the ray $m=\overrightarrow{P Q}$ will be important to our discussion. Let us refer to it as a closed cone $\bar{C}(\overrightarrow{P Q}, \alpha)$. We are particularly interested in the set $\bar{C}(\overrightarrow{P Q}, \alpha) \backslash\{P\}$, we call it a cone of angle $\alpha$ at $P$ (or rooted at $P$ ) and denote it by $C(\overrightarrow{P Q}, \alpha)$.

The usual Euclidean axioms of betweenness, incidence and angles hold in the hyperbolic case. Therefore two cones $C(m, \alpha)$ and $C(n, \beta)$ at $P$ do not intersect if and only if the angle between $m$ and $n$ is greater than $\alpha+\beta$. We say such pairs of cones are disjoint. 
Observation 1. Given any finite integer $d$ we can always construct d mutually disjoint cones at $P$ by taking d different rays and cones of suitably small angles around them.

Our goal is to compute an embedding function $\Phi: V \rightarrow \mathbb{H}$, where $V=v_{0}, v_{1}, v_{2}, \ldots$ is the set of vertices of the tree. To abbreviate notations, we write $\varphi_{i}=\Phi\left(v_{i}\right)$. We sometimes abbreviate our notations for cones as $C_{i j}^{\alpha}=C\left(\overrightarrow{\varphi_{i} \varphi_{j}}, \alpha\right)$.

We consider distortion over some set $W$ of pairs of distinct vertices in question. For example, $W$ can be the set of edges in the tree, or it can be the set of all pairs of vertices.

Distortion. We define the contraction factor over $W$ as $\delta_{c}=\max _{(i, j) \in W} \frac{\left|v_{i} v_{j}\right|_{T}}{\left|\varphi_{i} \varphi_{j}\right|_{\mathbb{H}}}$, and similarly the expansion factor as $\delta_{e}=\max _{(i, j) \in W} \frac{\left|\varphi_{i} \varphi_{j}\right|_{\mathbb{H}}}{\left|v_{i} v_{j}\right|_{T}}$. The distortion is defined as $\delta=\delta_{c} \cdot \delta_{e}$.

Observe that if the embedding globally scales all distances for pairs in $W$ by the same factor, then $\delta=1$.

We consider Voronoi diagrams in $\mathbb{H}$. Given a finite set of vertices $v_{0}, v_{1}, \cdots \subset$ $\mathbb{H}$, the Voronoi cell of $v_{i}$, denoted $\mathcal{V}\left(v_{i}\right)$ is the set of points whose distance to $v_{i}$ is not larger than the distance to $v_{j}$ for any $j \neq i$. The Delaunay Graph is its dual: given a set of vertices in $\mathbb{H}$ their Delaunay graph is one where a pair of vertices are neighbors if their Voronoi cells intersect. As with the euclidean case, this delaunay graph contains the MST.

Delaunay Embedding of Graphs: Given a graph $G$, its Delaunay embedding in $\mathbb{H}$ is an embedding of the vertices such that their Delaunay graph is $G$.

\section{Delaunay Embedding of Trees}

In this section, we describe the basic construct of embedding a tree as a Delaunay graph. The Delaunay graph automatically has the minimum spanning tree embedding and greedy embedding property. The basic idea is not new, and has been used in 9214 . But we wish to make it more general and write in terms of cones rooted at the embedded vertices. This makes it easier to handle weighted trees and low distortion embedding in following sections.

Reorganizing equation 1, we have that given rays $m_{+\alpha}$ and $m_{-\alpha}$ through $P$, the distance $|P Q|_{\mathbb{H}}$ to a point $Q$ on $m$ so that the line $l \perp P Q$ at $Q$ is limiting parallel to $m_{-\alpha}$ and $m_{+\alpha}$ is given by:

$$
|P Q|_{\mathbb{H}}=-k \ln \left(\tan \frac{\alpha}{2}\right) .
$$

Since $\alpha$ is always less than $\pi / 2$ radians, we have $\tan \frac{\alpha}{2}<1$. Therefore $|P Q|_{\mathbb{H}}$ is positive and monotone decreasing in $\alpha$. This means in particular, that if $|P Q|_{\mathbb{H}}$ is larger, then $l$ is limiting parallel to the bounding lines of a smaller cone, and therefore divergent parallel to $m_{-\alpha}$ and $m_{+\alpha}$, and fully contained in $C(m, \alpha)$.

We construct a function $\Phi$ that embeds the vertices of a tree $T$ into $\mathbb{H}$. Function $\Phi$ is designed such that $T$ is the Delaunay graph of the embdded vertices. 
Lemma 1. Given two cones $C(\overrightarrow{P R}, \alpha)$ and $C(\overrightarrow{R P}, \beta)$, and $\gamma=\min (\alpha, \beta)$, if $|P R|_{\mathbb{H}} \geq-2 k \ln \left(\tan \frac{\gamma}{2}\right)$ the perpendicular bisector of the segment $P R$ lies in the intersection $C(\overrightarrow{P R}, \gamma) \cap C(\overrightarrow{R P}, \gamma)$ which is contained in the intersection $C(\overrightarrow{P R}, \alpha) \cap C(\overrightarrow{R P}, \beta)$.

Proof. Suppose without loss of generality that $\gamma=\alpha$. Let us say that the perpendicular bisector $l$ intersects $P R$ at its midpoint $Q$.

Then $|P Q|_{\mathbb{H}} \geq-k \ln \left(\tan \frac{\alpha}{2}\right)$ and therefore $l \subset C(\overrightarrow{P R}, \alpha)$. Symmetrically, $|R Q|_{\mathbb{H}} \geq-k \ln \left(\tan \frac{\alpha}{2}\right) \Longrightarrow l \subset C(\overrightarrow{R P}, \alpha)$. Since $\alpha \leq \beta$, we have $C(\overrightarrow{R P}, \alpha) \subseteq$ $C(\overrightarrow{R P}, \beta)$. Therefore $l \subset C(\overrightarrow{P R}, \alpha) \cap C(\overrightarrow{R P}, \alpha)$ and $l \subset C(\overrightarrow{P R}, \alpha) \cap C(\overrightarrow{R P}, \beta)$.

This means in general we can consider the smaller of the two angles and consider the two cones to be of this same angle. See Figure 2. We say a segment $P R$ is Delaunay for angle $\gamma$ if it satisfies the conditions of the lemma for $\gamma=\alpha=\beta$.

Now we describe embedding an edge into a given cone. The goal is to embed in a way such that the Voronoi cell of one embedded vertex is completely contained inside a cone at the other vertex, and the edge is realized as the Delaunay edge between the embedded vertices, and the two cones can have the same angle. Again, see Figure 2,

Edge embedding function $\Phi$. Given an edge $v_{i} v_{j}$ and an angle $\alpha$, we select a cone $C(m, \alpha)$ at point $P$, and embed as follows. Vertex $v_{i}$ is embedded at $\varphi_{i}=P$. We select a point $R$ on the ray $m$ such that $|P R|_{\mathbb{H}} \geq-2 k \ln \left(\tan \frac{\alpha}{2}\right)$, and embed $v_{j}$ as $\varphi_{j}=R$. We call this a Delaunay embedding of the edge for angle $\alpha$.

This construction satisfies the conditions of lemma 1 with $\gamma=\alpha=\beta$, and therefore cones of angle $\alpha$ at $\varphi_{i}$ and $\varphi_{j}$ contain the perpendicular bisector of $\varphi_{i} \varphi_{j}$

Now we extend the embedding to the entire tree. The idea is to embed such that each edge is Delaunay for a suitable angle, and the corresponding cones rooted at a vertex are disjoint.

Definition 1. Tree Embedding Function $\Phi$. The vertices of a tree are embedded in a way that allows an assignment of an angle $\theta(e)$ to each edge e such that:

1. Cones determined by $\theta$ over all edges incident on any vertex are disjoint.

2. For any edge $v_{i} v_{j}$, its embedding $\varphi_{i} \varphi_{j}$ is Delaunay for angle $\theta\left(v_{i} v_{j}\right)$.

The following algorithm describes the construction of such a $\Phi$. Without loss of generality, we treat the input as a rooted tree.

Algorithm: Construction of $\Phi$ for $T$. Embed the root $v_{0}$ to an arbitrary point $\varphi_{0}$. If $v_{0}$ has $d$ children, they are Delaunay embedded individually into $d$ disjoint cones at $\varphi_{0}$. We embed all other vertices inductively as follows. Suppose 


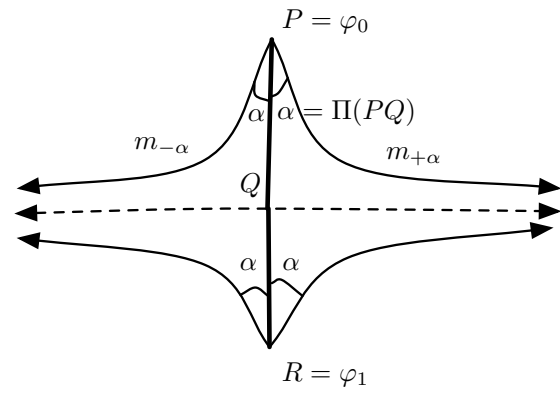

(a)

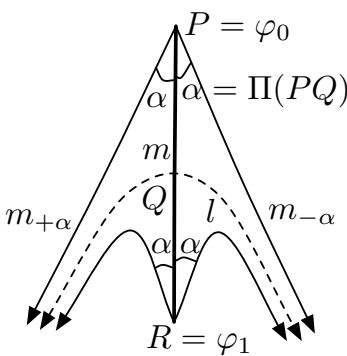

(b)

Fig. 2. Embedding an edge $v_{0} v_{1}$. (a) Shows the symmetry in the embedding - it is the same from the point of view of both end points. The Voronoi edge is the dashed line. (b) shows a different view, which is more akin to the view from point $P$. This view gives the intuition that we can embed any number of cones of small enough angles at $P$, and by symmetry, same is true at point $R$.

$v_{j}$ is a child of $v_{i}$, and has been embedded in a cone of angle $\alpha$ at $\varphi_{i}$. The children of $v_{j}$ are Delaunay embedded in mutually disjoint cones that are also disjoint from the cone $C\left(\overrightarrow{\varphi_{j} \varphi_{i}}, \alpha\right)$.

By construction, this algorithm produces an embedding that satisfies definition 1. The construction works for infinite trees as well.

Lemma 2. If $v_{i}$ is the parent of $v_{i+1}$, and $\Phi$ embeds the edge $v_{i} v_{i+1}$ in the cone $C\left(\overrightarrow{\varphi_{i}, \varphi_{i+1}}, \alpha\right)$ then

1. The Voronoi cells of all nodes in the subtree rooted at $\varphi_{i+1}$ are contained in $C\left(\overrightarrow{\varphi_{i}, \varphi_{i+1}}, \alpha\right)$.

2. The Voronoi cell of any node not in the subtree rooted at $\varphi_{i+1}$ are contained in the cone $C\left(\overrightarrow{\varphi_{i+1}, \varphi_{i}}, \alpha\right)$.

The hyperbolic plane contains many mutually parallel lines. Voronoi edges can be aligned to such lines, therefore, the Voronoi diagram consists of disjoint lines, carving out many disjoint half planes. The consequence is that Voronoi cells of nodes from different subtrees do not intersect. The only pairs of Voronoi cells that can intersect correspond to pairs of nodes that form edges of the tree. Therefore:

Theorem 2. The function $\Phi$ embeds $T$ as a Delaunay graph in $\mathbb{H}$.

This directly implies that $T$ is embedded as the minimum spanning tree of its vertices, since the Delaunay graph contains the MST.

Greedy Embedding. Greedy routing is a well studied problem in wireless networks. Delaunay graphs are significant to this problem as well: 


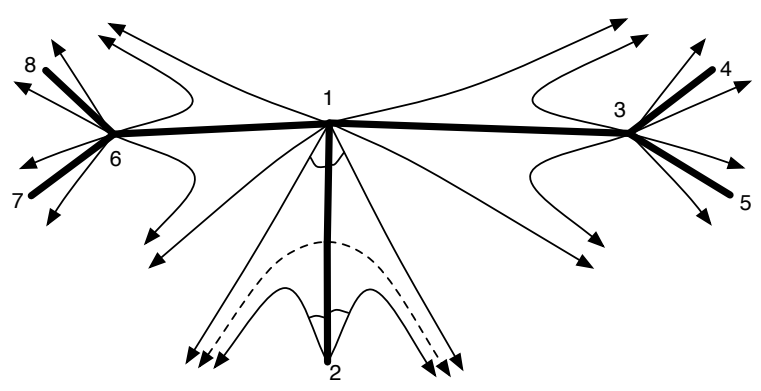

Fig. 3. Embedding a tree, by using cones of the type shown in Fig. 2 for each edge. $1,2,3 \ldots$ are the vertices of the tree. The rays form the cones. The thick edges are the edges of the tree.

Theorem 3. A Delaunay embedding guarantees delivery by greedy routing.

This implies the result of [9]. In fact the embedding of [9] is a specific instance of Delaunay embedding.

Edge Insertion. It is easy to adapt the algorithm to allow dynamic insertion into trees. At every vertex, we select the cones such that there is always enough angle left over to create more cones. This can always be done, since we can make the new cone small enough to not cover the entire available space. A new edge can be inserted into a new cone created in this space. This implies the edge insertion result of [2].

\section{Delaunay Embedding of Metric Trees}

Now we show a stronger embedding. Suppose $T=(V, E, w)$ is a weighted tree, where $w: E \rightarrow \mathbb{R}$ is the weight or length function on the edges. The goal is to realize the weight $w\left(v_{i} v_{j}\right)$ on each edge $v_{i} v_{j}$ as the length $\left|\varphi_{i} \varphi_{j}\right|_{\mathbb{H}}$ of the edge in the Delaunay embedding of the tree. For now, we are interested only in the distortion of individual edges of the tree, and show that in that sense there is an embedding with no distortion. The general distortion case is handled in the next section.

In the previous section we saw that each edge has to be embedded to a minimum length depending on the cone in which it is embedded. Based on this idea, we proceed as follows.

For each edge $e$, we compute a minimum length $L(e)$ needed to embed it as a Delaunay edge along with its neighboring edges. This gives a minimum scaling factor $\eta(e)=L(e) / w(e)$ for each edge. Next we compute $\eta_{\max }=\max _{e \in E} \eta(e)$ as the worst scaling needed at any edge. Then the tree is embedded such that each edge has length $\eta_{\max } \cdot w(e)$, that is, each edge is scaled by the same amount. Therefore, for unit length $\eta_{\max }$ the Delaunay graph realizes the metric tree. 
Algorithm: Computation of $\eta_{\max }$. Let us write $d(v)$ for the degree of a vertex $v$. The algorithm executes 5 passes over the tree:

1. Select for each vertex a maximum cone angle $\mu\left(v_{i}\right)<2 \pi / d\left(v_{i}\right)$.

2. Select for each edge $v_{i} v_{j}$ the maximum cone angle as $\alpha_{i j}=\min \left(\mu\left(v_{i}\right), \mu\left(v_{j}\right)\right)$.

3. Compute for each edge the minimum required length $L\left(v_{i} v_{j}\right)=-2 k \ln \left(\tan \frac{\alpha_{i j}}{2}\right)$.

4. Compute for each edge the minimum scaling factor $\eta\left(v_{i} v_{j}\right)=L\left(v_{i} v_{j}\right) / w\left(v_{i} v_{j}\right)$.

5. Compute the max value of $\eta$ over all edges : $\eta_{\max }=\max _{v_{i} v_{j} \in E} \eta\left(v_{i} v_{j}\right)$.

Algorithm: Embedding function $\Phi_{w}$ of metric tree $T$. The function is a special case of $\Phi$, and proceeds the same way. The cone construction and embedding steps are made more specific as follows:

1. At vertex $\varphi_{i}$ with parent $\varphi_{h}$, we create $d\left(v_{i}\right)-1$ disjoint cones of angle $\mu\left(v_{i}\right)$, disjoint from cone $C\left(\overrightarrow{\varphi_{i} \varphi_{h}}, \mu\left(v_{i}\right)\right)$.

2. A child $v_{j}$ of $v_{i}$ is embdded in the cone $C\left(\overrightarrow{\varphi_{i} \varphi_{j}}, \mu\left(v_{i}\right)\right)$ such that $\left|\varphi_{i} \varphi_{j}\right|_{\mathbb{H}}=$ $\eta_{\max } \cdot w\left(v_{i} v_{j}\right)$.

For any edge $v_{i} v_{j}$ in $E$, the function $\Phi_{w}$ embeds it in cones $C\left(\overrightarrow{\varphi_{i} \varphi_{j}}, \mu\left(v_{i}\right)\right)$ and $C\left(\overrightarrow{\varphi_{j} \varphi_{i}}, \mu\left(v_{j}\right)\right)$ respectively.

The need to compute $\eta_{\max }$ implies that algorithm works only on finite trees. But if constant bounds are known for the maximum vertex degree and the minimum edge length, then $\eta_{\max }$ can be computed beforehand and the algorithm can be applied to infinite trees.

Lemma 3. $\left|\varphi_{i} \varphi_{j}\right|_{\mathbb{H}} \geq-2 k \ln \left(\tan \frac{\alpha_{i j}}{2}\right)$.

Therefore, the embedding satisfies definition 1 and:

Theorem 4. The embedding $\Phi_{w}$ is a Delaunay embedding of a metric tree with distortion 1 over the set of edges of the tree.

\section{Delaunay Embedding with $(1+\varepsilon)$ Distortion: Hyperbolic Spanner}

In this section, we address the question of reducing the overall distortion to arbitrarily close to 1 . Thus, in such an embedding, the tree acts as hyperbolic spanner of the embedded points. This embedding also implies that known results that show existence of (tree, embedding) pairs translate to existence of embeddings in the hyperbolic plane.

Definition 2. $\beta$ separated cones. Suppose cones $C\left(\overrightarrow{\varphi_{j} \varphi_{i}}, \alpha\right)$ and $C\left(\overrightarrow{\varphi_{j} \varphi_{x}}, \gamma\right)$ are adjacent with the same root $\varphi_{j}$. Then the cones are $\beta$ separated if the two cones are an angle $2 \beta$ apart. That is, for arbitrary points $p \in C\left(\overrightarrow{\varphi_{j} \varphi_{i}}, \alpha\right)$ and $q \in C\left(\overrightarrow{\varphi_{j} \varphi_{x}}, \gamma\right)$, the angle $\angle p \varphi_{j} q>2 \beta$

An embedding is globally $\beta$ separated if every pair of adjacent cones used for embedding are $\beta$ separated. 
Lemma 4. If cones $C\left(\overrightarrow{\varphi_{j} \varphi_{i}}, \alpha\right)$ and $C\left(\overrightarrow{\varphi_{j} \varphi_{x}}, \gamma\right)$ are $\beta$ separated and $\varphi_{r} \in$ $C\left(\overrightarrow{\varphi_{j} \varphi_{i}}, \alpha\right)$ and $\varphi_{s} \in C\left(\overrightarrow{\varphi_{j} \varphi_{x}}, \gamma\right)$ then there is a constant $\nu$ depending only on $\beta$ such that $\left|\varphi_{r} \varphi_{j}\right|_{\mathbb{H}}+\left|\varphi_{s} \varphi_{j}\right|_{\mathbb{H}}>\left|\varphi_{r} \varphi_{s}\right|_{\mathbb{H}}>\left|\varphi_{r} \varphi_{j}\right|_{\mathbb{H}}+\left|\varphi_{s} \varphi_{j}\right|_{\mathbb{H}}-\nu$.

Proof. Suppose $l$ is the line limiting parallel to both the rays $\overrightarrow{\varphi_{j} \varphi_{r}}$ and $\overrightarrow{\varphi_{j} \varphi_{s}}$. Let us refer to the point $\varphi_{j}$ as $P$, and the perpendicular on $l$ as $P Q$. Then we know that $|P Q|_{\mathbb{H}} \leq-k \ln \left(\tan \frac{\beta}{2}\right)$.

Now, $\varphi_{j}, \varphi_{r}, \varphi_{s}$ are on the same side of $l$. Therefore, $\varphi_{r} \varphi_{s}$ intersects $P Q$, say at $W$. This implies that $P W<P Q$.

By triangle inequalities : $\left|\varphi_{r} \varphi_{j}\right|_{\mathbb{H}}+\left|\varphi_{s} \varphi_{j}\right|_{\mathbb{H}}>\left|\varphi_{r} \varphi_{s}\right|_{\mathbb{H}}>\left|\varphi_{r} \varphi_{j}\right|_{\mathbb{H}}+\left|\varphi_{s} \varphi_{j}\right|_{\mathbb{H}}-$ $2|P W|_{\mathbb{H}}>\left|\varphi_{r} \varphi_{j}\right|_{\mathbb{H}}+\left|\varphi_{s} \varphi_{j}\right|_{\mathbb{H}}-2|P Q|_{\mathbb{H}}$. Substituting $\nu=2|P Q|_{\mathbb{H}} \leq-2 k \ln \left(\tan \frac{\beta}{2}\right)$, we get the result.

If we can embed a tree such that neighboring edges, that is, edges incident on the same vertex, are always Delaunay in $\beta$ separated cones for some suitably small beta, then for such embeddings, we have the following theorem:

Theorem 5. If all edges of $T$ are scaled by a constant factor $\tau \geq \eta_{\max }$ such that each edge is longer than $\nu \frac{(1+\varepsilon)}{\varepsilon}$ and the Delaunay embedding of $T$ is $\beta$ separated, then the distortion over all vertex pairs is bounded by $1+\varepsilon$.

Proof. Let $v_{i}, v_{i+1}, v_{i+2}, \ldots, v_{i+p}$ be the path in the tree between the two end points. For any vertex $v_{i+j}$ on the path, observe that $\varphi_{i}$ is contained in a cone $C\left(\overrightarrow{\varphi_{i+j} \varphi_{i+j-1}}, \alpha\right)$ - a consequence of lemma 2 This cone, by construction, is $\beta$ separated from cone $C\left(\overrightarrow{\varphi_{i+j} \varphi_{i+j+1}}, \gamma\right)$ containing $\varphi_{i+j+1}$.

Thus we have $:\left|\varphi_{i} \varphi_{i+2}\right|_{\mathbb{H}} \geq\left|\varphi_{i} \varphi_{i+1}\right|_{\mathbb{H}}+\left|\varphi_{i+1} \varphi_{i+2}\right|_{\mathbb{H}}-\nu \geq\left|\varphi_{i} \varphi_{i+1}\right|_{\mathbb{H}}+$ $\left|\varphi_{i+1} \varphi_{i+2}\right|_{\mathbb{H}}-\frac{\varepsilon}{1+\varepsilon}\left|\varphi_{i+1} \varphi_{i+2}\right|_{\mathbb{H}}$, since each edge is longer than $\nu \frac{1+\varepsilon}{\varepsilon}$. Repeating, we have

$$
\begin{aligned}
\left|\varphi_{i} \varphi_{i+2}\right|_{\mathbb{H}} & \geq\left|\varphi_{i} \varphi_{i+1}\right|_{\mathbb{H}}+\left|\varphi_{i+1} \varphi_{i+2}\right|_{\mathbb{H}}-\frac{\varepsilon}{1+\varepsilon} \cdot\left|\varphi_{i+1} \varphi_{i+2}\right|_{\mathbb{H}} \\
\left|\varphi_{i} \varphi_{i+3}\right|_{\mathbb{H}} & \geq\left|\varphi_{i} \varphi_{i+2}\right|_{\mathbb{H}}+\left|\varphi_{i+2} \varphi_{i+3}\right|_{\mathbb{H}}-\frac{\varepsilon}{1+\varepsilon} \cdot\left|\varphi_{i+2} \varphi_{i+3}\right|_{\mathbb{H}} \\
\left|\varphi_{i} \varphi_{i+4}\right|_{\mathbb{H}} & \geq\left|\varphi_{i} \varphi_{i+3}\right|_{\mathbb{H}}+\left|\varphi_{i+3} \varphi_{i+4}\right|_{\mathbb{H}}-\frac{\varepsilon}{1+\varepsilon} \cdot\left|\varphi_{i+3} \varphi_{i+4}\right|_{\mathbb{H}} \\
& \cdots \\
\left|\varphi_{i} \varphi_{i+p}\right|_{\mathbb{H}} & \geq\left|\varphi_{i} \varphi_{i+p-1}\right|_{\mathbb{H}}+\left|\varphi_{i+p-1} \varphi_{i+p}\right|_{\mathbb{H}}-\frac{\varepsilon}{1+\varepsilon} \cdot\left|\varphi_{i+p-1} \varphi_{i+p}\right|_{\mathbb{H}}
\end{aligned}
$$

Adding:

$$
\left|\varphi_{i} \varphi_{i+p}\right|_{\mathbb{H}} \geq \sum_{x=i}^{i+p-1}\left|\varphi_{x} \varphi_{x+1}\right|_{\mathbb{H}}-\sum_{x=i}^{i+p-1} \frac{\varepsilon}{1+\varepsilon}\left|\varphi_{x} \varphi_{x+1}\right|_{\mathbb{H}}
$$

Therefore, $\left|\varphi_{i} \varphi_{i+p}\right|_{\mathbb{H}} \geq \frac{1}{1+\varepsilon} \cdot \sum_{x=i}^{i+p-1}\left|\varphi_{x} \varphi_{x+1}\right|_{\mathbb{H}}$. 
Since $\tau$ is fixed for all edges, $\left|\varphi_{i} \varphi_{i+p}\right|_{\mathbb{H}} \geq \frac{1}{1+\varepsilon} \cdot \tau\left|v_{i} v_{i+p}\right|_{T}$.

All edges are assumed to be scaled by a factor $\tau \geq \eta_{\max }$ such that they are longer than $\nu \frac{(1+\varepsilon)}{\varepsilon}$. We can therefore assume without loss of generality that $\tau>1$. Now, for vertices $v_{i}$ and $v_{j},\left|v_{i} v_{j}\right|_{\mathbb{H}} \leq \tau\left|v_{i} v_{j}\right|_{T}$. That is, hyperbolic distance is at most $\tau$ times the tree distance.

Thus the expansion factor $\delta_{e}$ is dominated by the edges of the tree satisfying equality, and $\delta_{e}=\tau$. The contraction factor is dominated by the pair with maximum distortion: $\delta_{c} \leq(1+\varepsilon) / \tau$. Therefore, distortion $\delta=\delta_{c} \cdot \delta_{e} \leq(1+\varepsilon)$.

Based on the theorem, the algorithm for $1+\varepsilon$ distortion embedding becomes simple:

Algorithm: $(1+\varepsilon)$ Distortion embedding. Suppose $d$ is the maximum degree of any node. Then a low distortion embedding algorithm follows in these steps:

1. Compute a cone separation angle $\beta<\pi / d$, and an angle for cones $\alpha=$ $2 \pi / d-2 \beta$. Set $\nu=-2 k \ln \left(\tan \frac{\beta}{2}\right)$.

2. Compute $\eta_{\max }$ as in the previous section.

3. Select $\tau>\eta_{\max }$ such that all edges are longer than $\nu \frac{1+\varepsilon}{\varepsilon}$.

4. Embed edges as before, but into $\beta$ separated cones, and edges scaled by a factor $\tau$.

The bounds of embedding arbitrary metrics into trees with low distortion extend to the hyperbolic plane via this result. From [6] we have that for any metric, there is a distribution over embeddings in the hyperbolic plane with expected distortion $O(\log n)$. Similarly 4] implies that for every graph there is an embedding into hyperbolic plane such that the average distortion of edges is bounded by $O\left(\log ^{2} n \log \log n\right)$. As before, the algorithm applies to infinite trees with known degree and length bounds.

\section{Conclusion}

We presented a method to embed trees into hyperbolic plane that simultaneously has several desirable properties. It is a Delaunay realization of the tree, preserves edge lengths exactly, and distance between non-neighbors is distorted by at most $1+\varepsilon$. This suggests hyperbolic plane as a useful general target to investigate embedding questions. It remains to be seen what additional properties can be obtained in $\mathbb{H}$, for the more general embedding questions.

Acknowledgement. Thanks to Jie Gao and the anonymous reviewers for many helpful comments on the original draft of the article. This work is funded by the German Research Foundation (DFG) through the research training group Methods for Discrete Structures (GRK 1408). 


\section{References}

1. Chepoi, V., Dragan, F., Estellon, B., Habib, M., Vaxès, Y., Xiang, Y.: Additive spanners and distance and routing labeling schemes for hyperbolic graphs. Algorithmica, 1-20 (2010), doi:10.1007/s00453-010-9478-x

2. Cvetkovski, A., Crovella, M.: Hyperbolic embedding and routing for dynamic graphs. In: Proceedings of Infocom 2009 (April 2009)

3. Dhamdhere, K., Gupta, A., Räcke, H.: Improved embeddings of graph metrics into random trees. In: Proceedings of the Seventeenth Annual ACM-SIAM Symposium on Discrete Algorithm, SODA 2006, pp. 61-69 (2006)

4. Elkin, M., Emek, Y., Spielman, D.A., Teng, S.-H.: Lower-stretch spanning trees. In: Proceedings of the Thirty-Seventh Annual ACM Symposium on Theory of Computing, STOC 2005, pp. 494-503 (2005)

5. Eppstein, D., Goodrich, M.T.: Succinct Greedy Graph Drawing in the Hyperbolic Plane. In: Tollis, I.G., Patrignani, M. (eds.) GD 2008. LNCS, vol. 5417, pp. 14-25. Springer, Heidelberg (2009)

6. Fakcharoenphol, J., Rao, S., Talwar, K.: A tight bound on approximating arbitrary metrics by tree metrics. In: Proceedings of the Thirty-fifth Annual ACM Symposium on Theory of Computing, pp. 448-455 (2003)

7. Greenberg, M.J.: Euclidean and Non-Euclidean Geometries. W.H. Freeman (1993)

8. Gromov, M.: Hyperbolic groups. In: Essays In Group Theory, pp. 75-263. Springer, New York (1987)

9. Kleinberg, R.: Geographic routing using hyperbolic space. In: Proceedings of the 26th Conference of the IEEE Communications Society (INFOCOM 2007), pp. 1902-1909 (2007)

10. Lee, J., Naor, A., Peres, Y.: Trees and markov convexity. Geometric and Functional Analysis 18, 1609-1659 (2009), doi:10.1007/s00039-008-0689-0

11. Monma, C., Suri, S.: Transitions in geometric minimum spanning trees (extended abstract). In: Proceedings of the Seventh Annual Symposium on Computational Geometry, pp. 239-249 (1991)

12. Papadopoulos, F., Krioukov, D., Boguñá, M., Vahdat, A.: Greedy forwarding in dynamic scale-free networks embedded in hyperbolic metric spaces. In: Proceedings of the 29th Conference on Information Communications, INFOCOM 2010, pp. 2973-2981 (2010)

13. Sarkar, R.: Low distortion delaunay embedding of trees in hyperbolic plane, http://page.inf.fu-berlin.de/sarkar/papers/HyperbolicDelaunayFull.pdf

14. Tanuma, T., Imai, H., Moriyama, S.: Revisiting hyperbolic voronoi diagrams from theoretical, applied and generalized viewpoints. In: International Symposium on Voronoi Diagrams in Science and Engineering, pp. 23-32 (2010)

15. Zeng, W., Sarkar, R., Luo, F., Gu, X.D., Gao, J.: Resilient routing for sensor networks using hyperbolic embedding of universal covering space. In: Proc. of the 29th Annual IEEE Conference on Computer Communications (INFOCOM 2010) (April 2010) 